\title{
Guest Editor's Foreword
}

It is my pleasure to present the Special Issue of Laser and Particle Beams devoted to the 25th anniversary of the Institute of High Current Electronics (IHCE) of the Russian Academy of Sciences Siberian Division.

The IHCE was established in Tomsk in 1977. The fact of its emergence formally marked the occurrence of a new field of science-high current electronics, which encompasses the studies in pulsed power, production and application of intense particle beams, physics of vacuum and gas discharges, and the interaction of intense energy flows with matter. The research and development work in the above individual areas had been performed at many scientific centers all over the world, and the IHCE pioneered a combined approach in studying all the relevant problems.

The successful 25-year history of the IHCE testifies that this choice of scientific strategy has proved itself. The Institute not only was able to preserve its potential in the hard post-Soviet time, when governmental support of Russian science had, in fact, ceased, but it became one of the world's leaders in the development of high-power pulse generators, particle accelerators, and X-ray sources. Widely known are the IHCE scientists' studies of high-current gas and vacuum discharges and work on modification of metal and dielectric surfaces using particle and plasma flows. The scientific equipment produced at the Institute is delivered to more than 10 countries. Among the regular partners of the Institute are national laboratories of the United States and leading scientific centers in France, Germany, and Japan.

The articles that constitute this Special Issue cover the whole spectrum of today's studies performed at the IHCE. There are several reviews of the work in some special areas, such as plasma electronics and production and application of intense low-energy electron beams, but the major part of the issue is formed by original articles that present the results obtained in 2002 .

The articles collected in one issue do not pretend to give a complete picture of the Institute's activities. I believe, however, that this publication will give the reader an adequate idea of the lines and standards of scientific research at the IHCE and will serve for further consolidation of the world's scientific community.

Gennady Mesyats Vice President of the Russian Academy of Sciences High Current Electronics Institute 4 Akademicheski Avenue Tomsk 634055, Russia 